\title{
Evaluation of the introduction of the one stop clinic and dedicated nurses for the ulcer cruris venosum care in the east of the Netherlands
}

\author{
Kroft EBM $^{1^{*}}$, Rook B ${ }^{1}$ and Wikkerink $\mathbf{M}^{2}$
}

Kroft EBM, Rook B, Wikkerink M. Evaluation of the introduction of the one stop clinic and dedicated nurses for the ulcer cruris venosum care in the east of the Netherlands. J Phlebol Lymphol. 2018;11(1):42-47.

Objective: In the Ziekenhuis Groep Twente (ZGT), a limited number of nurses from two community care organizations were trained by Dermatology nurses. These trained dedicated nurses visit the ulcer patients at home and give proper ulcer care. Furthermore the one stop clinic was introduced. The purpose of this study was to evaluate if there is a difference in time for wound healing for patients with UCV treated by dedicated nurses compared to treatment by nurses in the Dermatology outpatient clinic.

Methods: In this retrospective cohort study all the files of patients with an ulcer in the Dermatology department of ZGT in Almelo and Hengelo between 1 June 2010 and 1 June 2015 were studied.

Results: Out of a total of 385 new patients with an ulcer 97 patients were included. Patients who were treated by dedicated nurses were significant

\section{INTRODUCTION}

An ulcus cruris venosum (UCV) is the most prevalent cause of a leg ulcer. German studies show that the prevalence of general ulcer cruris in the general population is $1 \%$ and in individuals above 80 years between $4 \%$ and $5 \%$ [1]. Women are about three times more at risk of getting an ulcer compared to men [2]. Besides having an older age, there are other risk factors for UCV; these include varicose veins, vein abnormalities, multiple pregnancies, obesity, previous leg injuries (trauma), phlebitis, surgery and deep venous thrombosis (DVT) [3]. Leg ulcers can be divided into categories such as venous, arterial and venous-arterial, also known as mixed ulcers [4]. The majority of lower limb ulcers are caused by venous disease. Some ulcers can be caused by trauma, skin malignancies, inflammatory skin diseases (e.g. Necrobiosis lipoidica, scratch wounds by eczema), infections (e.g. bullous erysipelas) and more. The Lothian and Forth Valley Study showed that among 600 patients with ulcer cruris: $76 \%$ was caused by venous disease and $22 \%$ by arterial disease [5]. Concerning the mechanism of ulceration, or in other words the process of ulceration, pericapillary fibrin cuff formation, trapping of white blood cells and growth factors have been proposed as possible mechanisms of ulcerations [6]. Venous ulcers can cause various symptoms: pain, itching, heaviness, leg-tiredness, cramps, burning, and swelling [7]. Ulcers are debilitating and painful and can greatly reduce patients' quality of life [4]. Ulcer healing has been shown to restore quality of life [4].

The number of patients with UCV increase because of growing life expectancy and also more and more people develop obesity, often simultaneously. Moreover, since patients visit a hospital when the wound doesn't heal after a period of time, it is important to set the correct diagnosis as soon as possible. By setting a correct diagnosis, proper treatment can take place, resulting in a shorter time of wound healing and simultaneously cost reduction is expected. older $(\mathrm{p}=0.002)$ and had larger wounds $(\mathrm{p}=0.008)$. Age was not significantly related to time for wound closure (HR 0.99; $95 \%$ CI: 0.97-1.01, $\mathrm{p}=0.226$ ). After adjustment for confounders there was no significant difference in time for closure between dedicated nurses versus hospital care (HR 1.01; 95\% CI: 0.61-1.67, $\mathrm{p}=0.961$ ). The one stop clinic was statistically related to a shorter time of closure of the wound $(\mathrm{p}=0.009)$. The one stop clinic was also significant related to higher chance of wound closure (HR 1.94; 95\% CI: 1.15-3.27, $\mathrm{p}=0.013$ ).

Conclusions: This study showed that the time to wound closure is not statistical different between patients treated by dedicated nurses compared to hospital care when adjusted for the effects of the one stop clinic. The one stop clinic gives a significant better chance for faster time of wound closure controlled for both groups.

Key Words: Chronic leg ulcer; Ulcus cruris venosoum; Community care nursing; One stop clinic.

Most patients with an UCV are elderly, which requires significant effort for them to make frequent visits to the hospital. In the Ziekenhuis Groep Twente (ZGT), i.e. Hengelo and Almelo, the dermatologists and the community care organizations Carint Reggeland and ZorgAccent came up with a plan to train nurses of community care organizations by Dermatology nurses who daily practice the wound care at the outpatient Dermatology in the ZGT. A Canadian study from 2011 already showed that the organization of care rather than the location or way of care delivery is more important.(8) This is why we trained the nurses the same way of wound care as in the hospital. This collaboration was implemented by the Dermatology Department of the ZGT.

These trained community care nurses (dedicated nurses) visit the ulcer patients at home and give proper wound care and if necessary compression therapy. Patients can now be treated at home which makes the need for visits to the hospital no longer necessary. The quality of care is similar between care in a hospital setting and community care by dedicated nurses, only if the care is well organized [8,9]. Furthermore, at the ZGT the one stop clinic was introduced for faster stating a diagnosis and identifies which patients could be treated at home rather than the hospital setting. In one visit the medical history, physical examination and additional (vascular) examinations are performed. After this and still during the first visit, the diagnosis and the individual ulcer treatment plan are made. This new method is unique in the Netherlands. Although no much evidence is available about this subject yet, we suspect that patients prefer care at their homes, and that through this approach wounds would heal faster and makes this approach therefore more cost-efficient.

\section{Objectives}

We performed this retrospective study to investigate if there is a difference in time for wound healing for patients with UCV treated by dedicated nurses compared to treatment by nurses in a Dermatology outpatient

${ }^{1}$ Ziekenhuis Groep Twente, Zilvermeeuw 1, 7609 PP Almelo, The Netherlands

${ }^{2}$ Carintreggeland, Boortorenweg 20, 7554 RS Hengelo, The Netherlands

*Correspondence: Ilse B.M. Kroft, Ziekenhuis Groep Twente, Zilvermeeuw 1, 7609 PP Almelo, The Netherlands, Tel: +31612394407; E-mail: i.kroft@zgt.nl ; Doi: 10.14303/1983-8905.1000058

Received: May 25, 2018, Accepted: June 08, 2018, Published: June 15, 2018

open 2 access

This open-access article is distributed under the terms of the Creative Commons Attribution Non-Commercial License (CC BY-NC) (http:// creativecommons.org/licenses/by-nc/4.0/), which permits reuse, distribution and reproduction of the article, provided that the original work is properly cited and the reuse is restricted to noncommercial purposes. For commercial reuse, contact reprints@pulsus.com 
Clinic. Another aim of this study was to assess if the one stop clinic (faster diagnosis) lead to a faster time of wound closure?

\section{Diagnosis of ulcer cruris in the ZGT}

Besides the medical history and physical examination, additional examination will be performed. The tests which are used for the evaluation of the vascular functions are: the Ankle-Brachial Pressure Index (ABPI), duplex ultrasound and on indication Toe Brachial Index (TBI).

Diagnosis for superficial venosum reflux is available because of the duplex ultrasound scanning, which is the golden standard for accurate evaluation of the highest point of valve failure and extent of reflux $[10,11]$. That is the best way to confirm or exclude the presence of venous dysfunction. Duplex can determine whether the deep veins or the greater or lesser saphenous veins and their tributaries are dilated, congested, or incompetent. It can also detect the presence of incompetent perforating veins and if the problem is caused by anatomical obstruction, reflux, or both [12]. Venous incompetence was identified by reverse flow lasting $>0.5$ seconds following release of firm calf compression [13]. In the ZGT the most common tools for diagnosing ulcers are medical history, physical examination, vascular examination, like Duplex scanning, ABPI and TBI. Laboratory tests are sometimes performed as well, which could include blood testing or cultures.

The ABPI is the ratio of the systolic blood pressure measured at the ankle to that measured at the brachial artery [14]. However, the ABPI may not always be reliable, particularly in patients with diabetes where vascular calcification can prevent arterial compression and falsely increase arterial systolic pressure and therefore the ABPI [15]. The ABPI may thus be confounded in diabetic patients, because of calcification of the arteries. In that case the TBI can be performed [15].

After gathering all this information the dermatologist makes a diagnosis/ differential diagnosis. Sometimes additional tests are needed, like skin biopsy, blood samples and culture samples.

\section{Methods of treatment for UCV}

Treatment of patients with UCV is either conservative (compression therapy) or/and also with flebous intervention. In general, the goals of treatment are to reduce edema, improve ulcer healing, and prevent recurrence [12]. With appropriate treatment, most of these ulcers will heal within three to four months [16].

\section{Compression therapy}

Compression therapy aims to increase venous and lymphatic return, reducing oedema and venous pressure in the limb, by the application of an external force. Compression therapy is the standard of care for venous ulcers [17]. Compression treatment increases the healing of ulcers compared with no compression. More ulcers were healed with high compression systems than with low compression systems [18], but should only be used in the absence of significant arterial disease. No clear differences in the effectiveness of different types of compression systems (multilayer and short stretch bandages) have been shown. A meta-analysis showed that adequate compression therapy is not only important for the healing of venous insufficiency and ulcers but that continuing the compression after the ulcer has healed is also critical for the recurrencefree interval [19].

In addition to compression therapy, education is also important for both the healing process as to prevent recurrence i.e. inform the patient about the ulcer, the healing time and the importance of the treatments [20]. In addition to the UCV protocol the patient gets advised about their lifestyle: prevent immobility, stimulate to use the muscle calf pump, prevent obesity, and discourage smoking [21]

\section{Venous treatments}

Different techniques are available in the ZGT, including 'foam'sclerotherapy, endovenous radiofrequent ablation (VNUS) for the incompetent veins, and ambulant phlebectomy according to Muller.

\section{Wound care and wound care materials}

No level 1 evidence is available that any wound dressing confers specific benefit over another [12]. In the ZGT, alginate dressings, hydrofibers, hydrocolloids, hydrogels, wound edge protectors, foam and silver, paraffin and silicon dressings were used.

\section{Ulcer care ZGT}

The care path of patients with UCV before implementation of the dedicated nurses and the one stop clinic, is illustrated in Figure 1.

Until the year 2014 ZGT Almelo was using this "old method" and until the year 2004 ZGT Hengelo was using this "old method".

Patients with an UCV first went to the General Practitioner (GP). If the wound did not heal, or when the GP had doubt about the diagnosis, the patient was referred to the hospital. After multiple outpatients visits the diagnosis can be made (Figure 1). After the diagnosis, a treatment plan was prepared by the dermatologist. After this, the patient has to visit the hospital each time for the treatment and care of the ulcer wound by the dermatologist (nurse).

\section{Ziekenhuis Groep Twente (ZGT) introduction of the one stop clinic and the dedicated nurses}

The New method might be more time-saving by doing the different kinds of examinations and making the treatment plan during the first consult in the hospital. This is expected to save time because of less necessary consultations and thereby also less waiting time compared with the old method.

\section{One stop clinic}

Since 1997 the Dermatology outpatient clinic of ZGT cooperates with nurses who are dedicated in taking care of ulcer patients. Since the year of 2014 during the first consultation at the hospital, every ulcer patient receives: a medical history, physical examination, additional examination i.e. duplex ultrasound and ABPI and on indication TBI, laboratory tests and/or biopsy. After performing this, the diagnosis and the individual ulcer treatment plan are made. Information about the patient is (after informed consent is secured) exchanged with the dedicated nurses. Then information about the ulcer is given to the patient and a letter about the current state of the patient, about the ulcer and the treatment plan is sent to the GP.

Below in Figure 2 the one stop clinic is illustrated in more detail.

If the diagnosis during the first consult in the hospital shows that the ulcer is of venous origin, then two options of treatment are possible:

1. Conservative treatment: No treatment is possible, for example the patient has deep venous insufficiency (Figure 2). In this case only the intake (medical history and physical examination) and vascular examination are performed by the dermatologist. Immediately after that the patient is referred back to the GP and the dedicated nurses are activated. The GP becomes main therapist again.

2. Venous treatment: In case of superficial venous insufficiency (Figure 2). In this case the patient still remains under the supervision of the dermatologist and is also controlled by the dermatologist. This remains until venous intervention of the underlying cause has been performed by the dermatologist. Then the patient will be referred back to the GP. The GP becomes main therapist again and the dedicated team is activated. If the state of the UCV becomes worse, for example larger, then the dedicated nurse can contact the dermatologist directly. 


\section{The nurse team with a dedicated nurse}

Specific education and qualification requirements are required for a nurse to become a dedicated nurse.

\section{The education requirements are:}

- To become a certified dedicated wound nurse, a nurse must follow a training that consists of eight sessions with an experienced dedicated nurse and two sessions at the ZGT with a dermatology nurse of the ZGT and with the dermatologist.

- Also the nurse must receive three sessions of theoretical training: two sessions (accredited by the dedicated nurse) and one session phlebological education by a dermatologist.

- The team of nurses is trained twice a year by a dedicated nurse (for ulcers) from CarintReggeland and once a year an education course by a dermatologist of ZGT.

- The team has to participate two times a year in patient discussions.

- The education level of a nurse (for treating ulcers) must at least be level four of the secondary vocational education.

- The dedicated nurses must spend at least ten hours a week on ulcer treatment and/or compression therapy.

\section{The dedicated nurse in practice}

Dedicated nurses are especially trained to treat ulcer patients with wound care and compression therapy. Because of these dedicated nurses, the ulcer patients do not have to go to the hospital to be taken care of, but instead these dedicated ulcer nurses go to the patients' home. Usually a dedicated nurse visits the patients at home twice a week, but if necessary more often. The dedicated ulcer nurses have close communication with the dermatologist and nurses in the ZGT and with the GP. The nurses of ZGT, dedicated nurses of Carint Reggeland and ZorgAccent cooperate together and use the same type of wound material to bind and clean the wound. So when in a case a nurse from Carint Reggeland has to take over from a nurse from ZorgAccent or vice versa, then this can be done without problems. Also currently there is more economical use of wound materials. As an example previously leg bandages would be used once and then they would be thrown away. Nowadays wound bandages can be washed and reused again for two or three times. Furthermore, the nurses also work according to the same guidelines, such as the way to bind wounds and which wound products to use. They also report their data according to standardized dossier compilation.

\section{Summary of the advantage of one stop clinic and the dedicated nurses}

In summary, the advantage of the one stop clinic and the dedicated nurses team toward the old method of care is that the whole care path from the beginning till the end takes less time, there is better communication between the nurses, there are special working guidelines and standardized medical records compilation for reporting data and there is more economical use of wound care.

\section{Research questions}

\section{Aims of this study}

- Is the time of wound healing for patients with UCV faster with the deployment of dermatologist nurses in the hospital compared to treatment of dedicated nurses?

- Does the one stop clinic (faster diagnosis) lead to a faster time of wound closure?

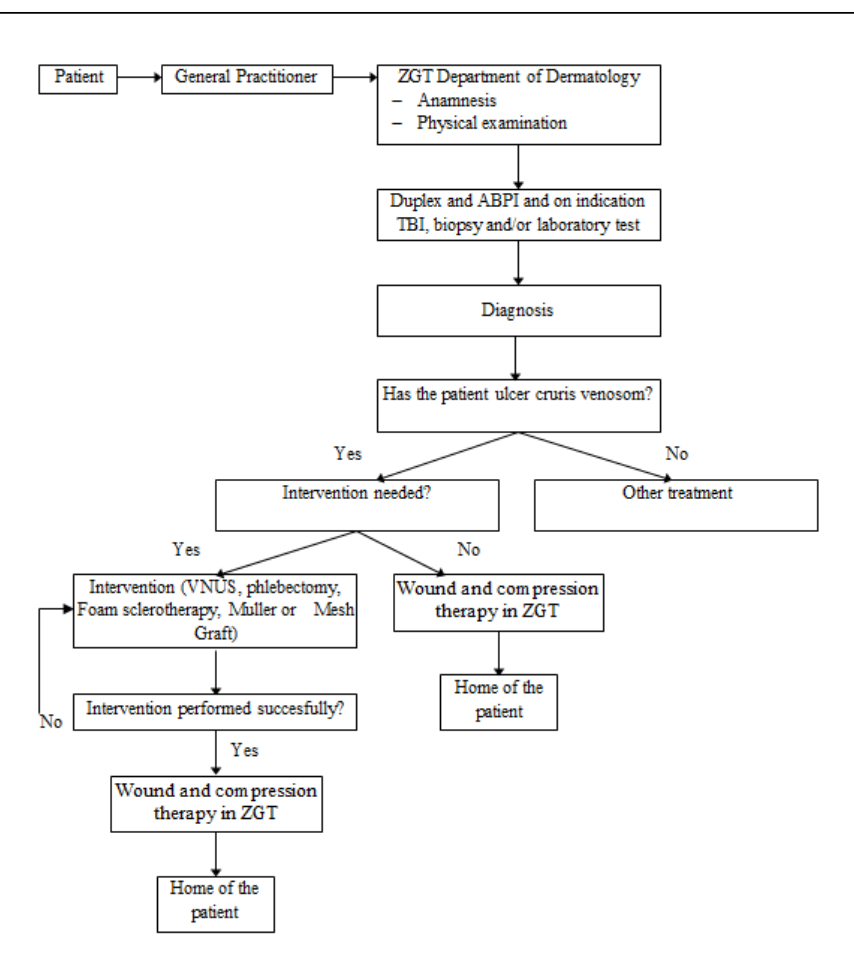

Figure 1: Old method. More visits were needed before a diagnosis was made. The patient receives care in the hospital (ABPI: ankle-brachial pressure index; TBI: toe brachial index; VNUS: endovenous radiofrequent ablation).

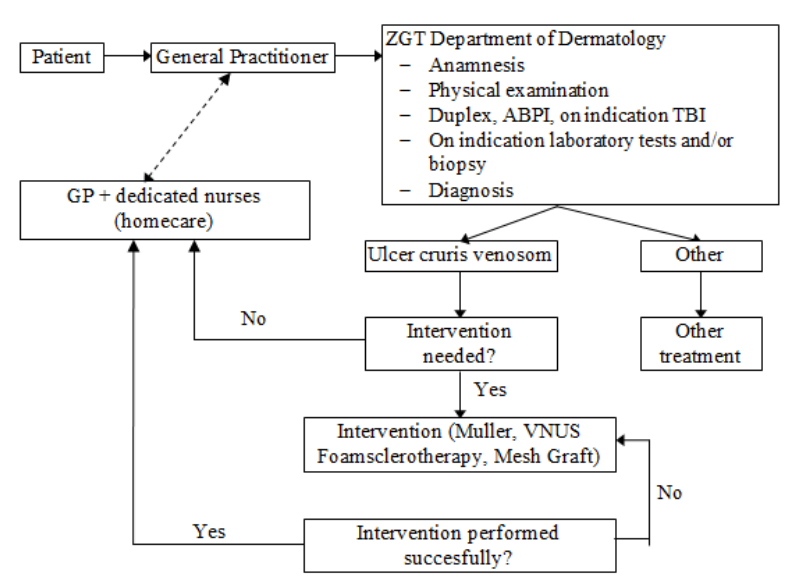

Figure 2: Overview of the one stop poli. During the "First consult" the anamneses, physical examination, vascular research and the diagnosis are performed in one day. Sometimes when laboratory test and/or biopsy are taken, the diagnosis comes later.

\section{METHODS}

\section{Study design}

This research study is a retrospective cohort study.

\section{Setting}

All the files of patients with a leg ulcer examined in the Dermatology department of ZGT in Almelo and Hengelo between June 1st 2010 and June 1st 2015 were studied. After this, we 385 patients were selected (Figure 3). 


\section{Participants}

Patients were included when they had an UCV and were eighteen years or older.

Exclusion criteria were patients with other causes of leg ulcer, e.g. arterial leg ulcers, ulcer of Martorell, livedovasculopathy, mixed ulcer: ulcer has both arterial and venous cause. Furthermore, patients with an ankle pressure $<60 \mathrm{~mm} \mathrm{Hg}$ and other causes of ulcers that do not have only a venous cause were excluded. Also patients under the age of eighteen and also mentally disabled patients (for example dementia) were not included in the research study. Of patients with an ulcer on both legs, the largest ulcer was included for analysis. Other exclusion criteria were patients treated by family/GP or other community care organizations after visiting the ZGT (one stop or no one stop).

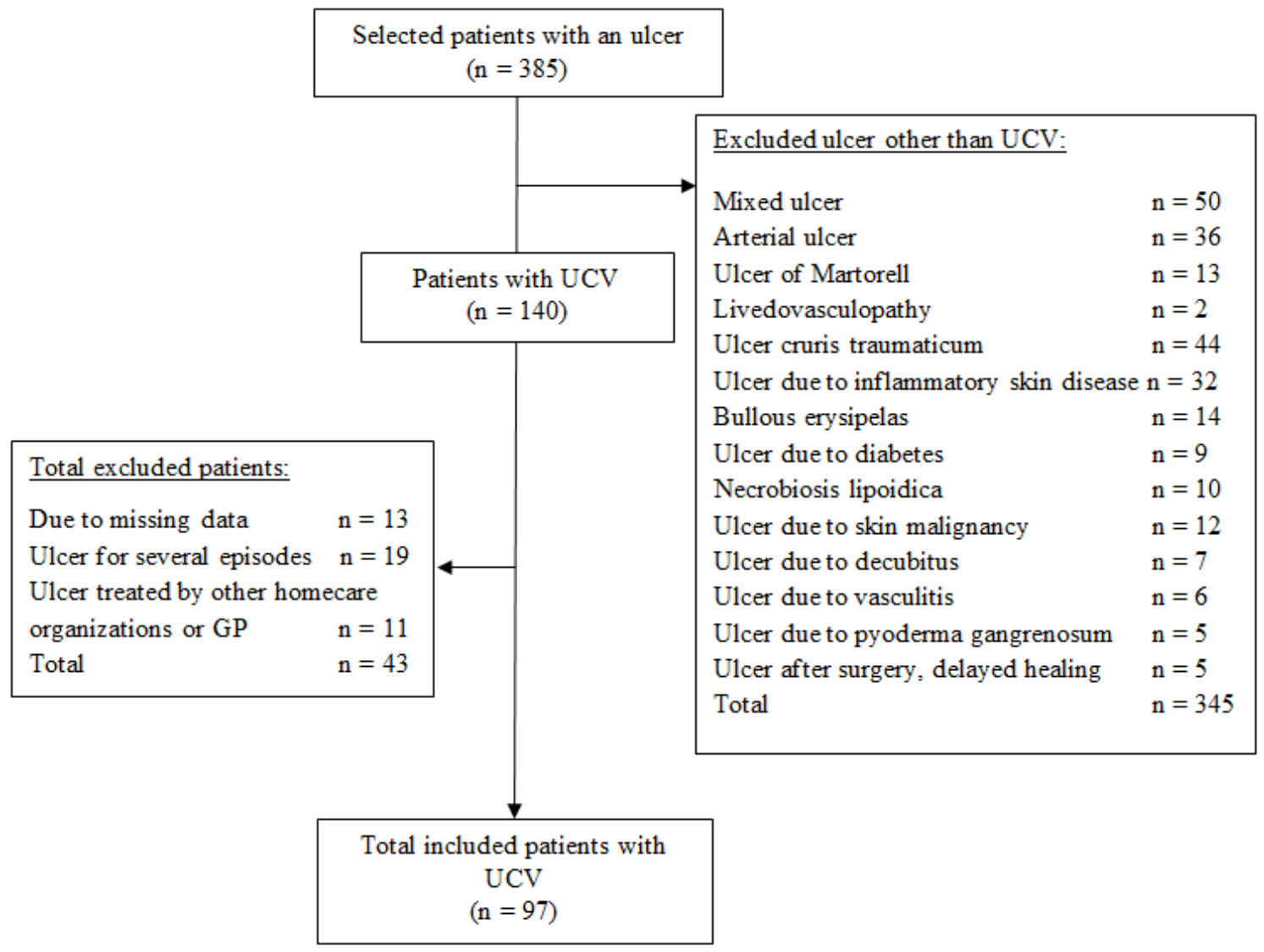

Figure 3: Patient selection (UCV: Ulcer cruris venosum; GP: General Practitioner).

\section{Study variables}

All files were searched and the following variables were identified: patient number, sex, date of birth, date of first visit, age at first visit, number of visits to the hospital, number of visits to a certain caretaker, ulcer side, ulcer size, type of ZGT method, type of treatment, state of closure of the wound, date of wound closure, first and last date of community care, number of treatments by community care and number of dedicated nurses at home.

\section{Patient groups}

The patients were assigned to groups based on clinical decision. There are the following groups of patients belonging to different kind of "care methods":

1. Patients who were treated via the one stop clinic and treated with the dedicated nurses (One stop+DDN) (New method)

2. Patients who were treated on the one stop clinic and were treated only in the ZGT, therefore no participation of the dedicated nurses (one stop+ZGT) (Transitional phase old and new method)

3. Patients who were treated not on the one stop clinic, but by the dedicated nurses (no one stop+DDN) (Transitional phase old and new method)
4. Patients who were treated not on the one stop clinic and were treated only in the ZGT (no one stop+ZGT) (Old method)

To compare dedicated nurses to ulcer care in the hospital we combined group 1 and 3 and group 2 and 4 due to small groups.

\section{Primary outcome variables}

The first primary outcome variable is time for wound healing for patients with UCV treated by dedicated nurses compared to treatment by nurses in a hospital setting.

The second primary outcome is the time to wound closure that may be saved by introducing the one stop clinic compared to the "old method".

\section{Statistical methods}

Continuous variables are expressed as mean with standard deviation (SD) or median with interquartile range (IQR); categorical variables as counts with corresponding percentages.

Difference in baseline characteristics between groups was tested using independent t-test or Mann-Whitney U test (continuous variables) or chisquare (categorical). We analyzed time to wound healing by Kaplan-Meier survival curves. 
We used univariate and multivariate Cox proportional hazard regression models to establish the relationship of treatment (ZGT vs. DDN) with wound healing.

Baseline characteristics that showed significant differences between groups were further analyzed using univariate Cox proportional hazard regression to explore potential confounding effects on the relation between treatment group and time to wound healing. A p-value of 0.150 or lower was included in the multivariate cox regression model.

\section{RESULTS}

\section{Patient characteristics}

A total of 97 cases were included. Group 1: $\mathrm{n}=26$ (male 12), group 2: $\mathrm{n}=16$ (male 8), group 3: $\mathrm{n}=23$ (male 8 ), group 4: $\mathrm{n}=32$ (male 15). To compare dedicated nurses to ulcer care in the hospital we combined group 1 and 3, named DDN and group 2 and 4, named ZGT, due to small groups.

Of these 97 cases, (male $n=43$, female $n=45$ ) with median age 70.6 years, (interquartile range 23.7, minimum 30.4, maximum 93.6 years) the median ulcer size was $2.0 \mathrm{~cm}^{2}$ (interquartile range $3.5 \mathrm{~cm}^{2}$, minimum 0.01 $\mathrm{cm}-165.0 \mathrm{~cm})$, the median time to wound closure was 2.4 months $(95 \% \mathrm{CI}$ 1.9-2.9 months).

The patients who were treated by dedicated nurses were significant older $(\mathrm{p}=0.002)$ and had larger wounds $(\mathrm{p}=0.008)$. Age was not significantly related to time to wound closure (HR 0.99; 95\% CI: 1.0 (0.97)-1.0, $\mathrm{p}=0.226$ ).

Characteristics of both groups are shown in table 1. There was a significant difference between the DDN group and the ZGT group in median age at first visit $(\mathrm{p}=0.002)$ and in median ulcer size $(\mathrm{p}=0.008)$.

\section{Primary results}

The median time to wound closure was 2.2 months (interquartile range 1.4-3.0) in the DDN group and 2.3 months (interquartile range 2.1-2.6) in the ZGT group $(\mathrm{p}=0.688)$

The one stop clinic was statistically related to a shorter time to closure of the wound $(\mathrm{p}=0.009)$.

The median time to wound closure was 2.3 months, when no venous intervention occurred, with venous intervention, 2.2 months and with a meshgraft 4.8 months.

Table 1: Characteristics of both groups.

\begin{tabular}{|c|c|c|c|}
\hline Variable & DDN & ZGT & p-value \\
\hline Total, n (\%) & $49(100)$ & $48(100)$ & \\
\hline $\operatorname{Sex}(M / F), n(\%)$ & $20(41) / 29(59)$ & $23(48) / 25(52)$ & 0.482 \\
\hline $\begin{array}{l}\text { Median age at first visit, y } \\
\text { (IQR) }\end{array}$ & $75.6(16.8)$ & $65.5(24.5)$ & 0.002 \\
\hline Median ulcer size ${ }^{*}, \mathrm{~cm}^{2}(\mathrm{IQR})$ & $2.6(8.0)$ & $1.0(2.5)$ & 0.008 \\
\hline One stop, n (\%) & $26(53)$ & $16(33)$ & 0.05 \\
\hline \multicolumn{4}{|c|}{$\begin{array}{l}\text { M: Male; F: Female; IQR: Interquartile Range; } P \text {-values that are significant } \\
(p<0.05) \text { are reported bold. "The size of the wound was not known in all } \\
\text { patients: DDN } n=40, Z G T n=37\end{array}$} \\
\hline
\end{tabular}

The median time for wound healing was not statistically different between patients who had a venous intervention $(\mathrm{p}=0.290)$ or mesh graft $(\mathrm{p}=0.121)$ and patients without any of these interventional treatments.

There was no significant difference between both groups and the number of venous/mesh graft treatments.

\section{Secondary results}

Multivariable adjusted risk prediction:

After adjustment for confounders there was no significant difference in time to wound closure between dedicated nurses versus hospital care (HR 1.2; $95 \%$ CI: $0.6-2.1, p=0.623)$. One stop clinic was significant related to the time to wound closure (HR 2.3; 95\% CI: 1.3-4.2, $\mathrm{p}=0.005$ ). Size of ulcer was not related to the time to wound closure (HR $0.8 ; 95 \% \mathrm{CI}$ : $0.7-1.0, \mathrm{p}=0.054)$.

The purpose of this first retrospective study was to investigate if there is difference in effectiveness in terms of timesaving: time for wound healing for patients with UCV treated by dedicated nurses compared to treatment by nurses in a hospital setting.

The answer to this question is that there was no difference in time of wound healing between these two groups. Eg. time to wound closure was not statistical different between patients treated by dedicated nurse compared to hospital care when adjusted for confounders. This finding is interesting. Most patients were old, for whom we assume it could be difficult to go to a hospital. Care inside their own house gives more relief for the patients. Since there was no difference in time to wound closure, this suggests that the care can safely be arranged by dedicated nurses, which could be a benefit for these patients.

The patients who were treated by dedicated nurses were significant older and had larger wounds. Overall these patients were often not treated with venous intervention; due to comorbidity. Age at first visit and the size of the wounds did not confound results to wound closure time.

In conclusion, there was no significant difference in the time of wound healing between those treated by the dedicated nurses and the experienced nurses in the hospital.

The second purpose of this study was to examine if the one stop clinic leads to faster time to wound closure.

In this retrospective study, we found that the one stop clinic gives a significant shorter time to wound closure. This may be explained by the fact that while during the first visit to this one stop clinic duplex and arterial investigations were performed, sometimes additional laboratory testing and of a biopsy were needed. In the past, the additional tests were not always performed or these tests were performed when the ulcer did not heal in a short period of time. Because of this, there was some delay in the proper wound treatment. Nowadays, the correct diagnosis can mostly be stated during first visit, so the correct treatment was given directly.

The median time for wound healing was not statistically different between patients who had a venous intervention or mesh graft and patients without any of these treatments. This finding can be explained as being due to a limited number of patients.

\section{DISCUSSION}

\section{Key results}

The first outcome of this study is the affectivity in terms of timesaving: Is the time for wound healing for patients with UCV treated by dedicated nurses shorter, longer or equal compared to treatment by nurses in a hospital setting? This study showed that the time to wound closure not statistical different is between patients treated by dedicated nurse compared to hospital care when adjusted for the effects of the one stop clinic.

The second outcome involves: does the one stop clinic (faster diagnosis) lead to a faster time of wound closure? The one stop clinic gives a significant better chance for faster time of wound closure controlled for both groups.

\section{Limitations}

Between June $1^{\text {st }} 2010$ and June $1^{\text {st }} 2015,385$ patients with a new ulcer were seen in the hospital. Only 140 out of 385 patients had an UCV. The 
reason of this would be the strict inclusion criteria that were applied. Only patients with an ulcer cruris venosum were included. Because of the diverse pathways of care that patients could follow in the previous years, four different patients groups were formed. These reasons led to the small numbers of patients in each group, making it difficult to compare the groups. Since the old method is not used anymore the groups would not get bigger. Therefore in the analysis the groups were combined.

Analyzing the size of the wound was done by estimating the wounds on sight of a picture since in many cases a tape-measure was not used. Also the wound size was only measured two dimensionally; consequently the depth of the wound was not taken into account. However, since all wounds were assessed by the same person, we expect no great variations.

Besides the time for wound healing, we planned to identify the costs of treating the ulcers as well to compare the total amount of costs in these patients treated in the setting of hospital care, community care organizations and dedicated nurses. Information like number of visits, the amount of wound dressings that were used, the number of times gauze was switched and the time (dedicated) nurses spent at the patients home would all be gathered. However, while the information systems of the community care organization were not yet equipped to collect such data, the information was not documented correctly in the records and was therefore limited. Consequently in this retrospective study we could not analyze this data accurately. In our daily practice, we find that dedicated nurses, through their extensive knowledge, are much more cost effective on materials such as gauze, bandages and wound dressings. Identification of the costs of ulcers treated by dedicated nurses compared to the old method would allow health care workers and organizations to be more cost efficient in the care of ulcer. The total cost of ulcer care should be targeted for further research in an extensive multicenter trial. This way a reduction of costs in ulcer care could be achieved.

We assume that people are more satisfied while receiving treatment at home. However, the patient satisfaction has not been addressed in this study and future studies should examine the impact of preference of patients of the way of care on outcome. Furthermore, due to cost efficiency in the health care nowadays, patients would see a lot of different nurses treating their wounds. In most cases where dedicated nurses were the main therapist, they would be the only one treating each patient. This could increase the patients satisfaction. We therefore suggest further research which includes patient satisfaction, preference of treatment and quality of life.

\section{Interpretation}

This kind of study had not been performed previously. To our knowledge this is the first study that shows that a one stop clinic for ulcer cruris patients is very valuable. Firstly the one stop clinic gives a significant better chance for faster time of wound closure, because a proper diagnosis is made and a correct treatment can be started directly. This will result in lower costs for these ulcer treatments. Secondly by means of dedicated nurses, the treatment can be arranged at home as good as in the hospital, which must be a benefit for the mostly older patients.

\section{ACKNOWLEDGEMENTS}

The authors want to thank J. Timmerman, M. Vermeer, PhD (both Epidemiologists of the ZGT), R. Wijnsma (medical assistent Dermatology ZGT), M. Huis in 't Veld and A. Broekmaat (nurses Dermatology ZGT) and M. Beekman (dedicated nurse ZorgAccent) for their help in collecting and analyzing the data of the files.

\section{CONFLICT OF INTEREST}

This research was not sponsored.

\section{REFERENCES}

1. Wissing U, Unosson M. The relation between nutritional status and physical activity, ulcer history and ulcer-related problems in patients with leg and foot ulcers. Scand J Caring Sci. 1999;13:123-8.

2. Callam M, Ruckley C, Harper D, et al. Chronic ulceration of the leg: extent of the problem and provision of care. Br Med J. 1985; 290:1855-6

3. Agale S. Chronic Leg Ulcers: Epidemiology, Aetiopathogenesis, and Management. Ulcers. 2013;2013:9.

4. London N, Donnelly R. ABC of arterial and venous disease: Ulcerated lower limb. BMJ. 2000; 320:1589-91.

5. Callam M, Harper D, Dale JaRC. Chronic ulcer of the leg: clinical history. Br Med J. 1987;294:1389-91.

6. Falanga V, Eaglstein WH. The "trap" hypothesis of venous ulceration. Lancet. 1993;341:1006-8.

7. Khachemoune A, Kauffman C. Diagnosis of Leg Ulcers. Internet J Dermatol. 2001;1.

8. Harrison MB, VanDenKerkhof E, Hopman WM, et al. Evidenceinformed Leg Ulcer Care: A Cohort Study Comparing Outcomes of Individuals Choosing Nurse-led Clinic or Home Care. Ostomy Wound Manage. 2011;57:38-45.

9. Harrison MB, Graham ID, Lorimer K, et al. Nurse clinic versus home delivery of evidence-based community leg ulcer care: A randomized health services trial. BMC Health Serv Res. 2008;8:243.

10. Maessen-Visch MB. Diagnostiek en behandeling van het ulcus cruris venosum. Utrecht: Het Kwaliteitsinstituut voor de Gezondheidszorg CBO; 2005

11. Whiddon LL. The treatment of venous ulcers of the lower extremities. Proc (Bayl Univ Med Cent). 2007;20:363-6.

12. Van Gent BW, Wilschut E, Wittens C. Management of venous ulcer disease. Br Med J. 2010;341.

13. Dix FP, Picton A, McCollum CN. Effect of Superficial Venous Surgery on Venous Function in Chronic Venous Insufficiency. Ann Vasc Surg. 2005;19:678-5.

14. Aboyans V, Criquie M, Abraham P, et al. Measurement and interpretation of the Ankle-Brachial Index: a scientific statement from the American Heart Association. Circulation. 2012;126:2890-909.

15. Spangeus A, Wijkman M, Lindstrom T, et al. Toe brachial Index in middle aged patients with diabetes mellitus type 2: Not just a peripheral issue. Diabetes Res Clin Pract. 2013;100:195-202.

16. http://www.nhs.uk/Conditions/Leg-ulcer-venous/Pages/ Introduction.aspx

17. Fletcher A, Cullum N, Sheldon TA. A systematic review of compression treatment for venous leg ulcers. BMJ. 1997;315:576-80.

18. Cullum N, Nelson EA, Fletcher AW, et al. Compression for venous leg ulcers. Cochrane Database Syst Rev. 2004;11:CD000265.

19. Nelson EA, Bradley MD. Dressings and topical agents for arterial leg ulcers. Cochrane Database Syst Rev. 2007;1.

20. Van Hof N, Balak FSR, Apeldoorn L, et al. NHG-Standaard Ulcus cruris venosum. 2010;53:321-33.

21. Maessen-Visch M. Samenvatting Richtlijnen Dermatologie 2015. 